\title{
osmar: OpenStreetMap and $\mathbf{R}$
}

by Manuel J. A. Eugster and Thomas Schlesinger

\begin{abstract}
OpenStreetMap provides freely accessible and editable geographic data. The osmar package smoothly integrates the OpenStreetMap project into the R ecosystem. The osmar package provides infrastructure to access OpenStreetMap data from different sources, to enable working with the OSM data in the familiar $\mathrm{R}$ idiom, and to convert the data into objects based on classes provided by existing $\mathrm{R}$ packages. This paper explains the package's concept and shows how to use it. As an application we present a simple navigation device.
\end{abstract}

\section{Introduction}

"OpenStreetMap creates and provides free geographic data such as street maps to anyone who wants them" announces the OpenStreetMap wiki main page (OSM Foundation, 2011) - and we think R users want free geographic data. Therefore, the add-on package osmar (Schlesinger and Eugster, 2012) provides extensible infrastructure for integrating the OpenStreetMap project (OSM) into the R project.

The aim of the OpenStreetMap project is to create a free editable map of the world. The project maintains a database of geographic elements (nodes, ways and relations) and features (such as streets, buildings and landmarks). These data are collected and provided by volunteers using GPS devices, aerial imagery, and local knowledge. The most prominent application is the rendering of the geographic data and features into raster images (for example, for the OSM map on the website). However, the project also provides an application programming interface (API) for fetching raw data from and saving to the OSM database.

The OpenStreetMap project provides data in the OSM XML format, which consists of three basic elements:

Node: The basic element. It consists of the attributes latitude and longitude.

Way: An ordered interconnection of nodes to describe a linear feature (e.g., a street). Areas (e.g., buildings) are represented as closed ways.

Relation: A grouping of elements (nodes, ways, and relations), which are somehow geographically related (e.g., bus and cycle routes).

Each element has further attributes like the element ID (unique within the corresponding element group) and timestamp. Furthermore, each element may have an arbitrary number of tags (key-value pairs) which describe the element. Ways and relations, in addition, have references to their members' IDs.

In order to access the data, OSM provides an application programming interface (API) over the hypertext transfer protocol (HTTP) for getting raw data from and putting it to the OSM database. The main API (currently in version 0.6) has calls to get elements (and all other elements referenced by it) by, among other things, their ID and a bounding box. However, the requests are limited (e.g., currently only an area of 0.25 square degrees can be queried). An (unlimited) alternative is provided by planet files. These are compressed OSM XML files containing different OSM database extracts (e.g., the entire world or an individual country or area). Planet files can be downloaded from the OSM wiki and processed using the command-line Java tool Osmosis (Henderson, 2011).

For a complete introduction into the OSM project, the OSM API, and the OSM XML file format we refer to the project's wiki available at http://wiki .openstreetmap.org/.

The aim of the package osmar is to provide extensible infrastructure to get and to represent the above described OSM data within R, to enable working with the OSM data in the familiar R idiom, and to convert the OSM data to objects based on classes provided by other packages. Figure 1 visualizes the package's concept. This is a different idea than existing packages like OpenStreetMap (Fellows, 2012), RgoogleMaps (Loecher, 2012), and ggmap (Kahle and Wickham, 2012) follow. Whereas these packages provide access to the already rendered data (i.e., raster images), osmar enables the usage of the raw OSM data.

In the following section we present the package's implementation and usage. Note that we try to increase readability by only showing the relevant arguments of plot statements. We refer to the "navigator" demo in the osmar package for the actual plot statements. 


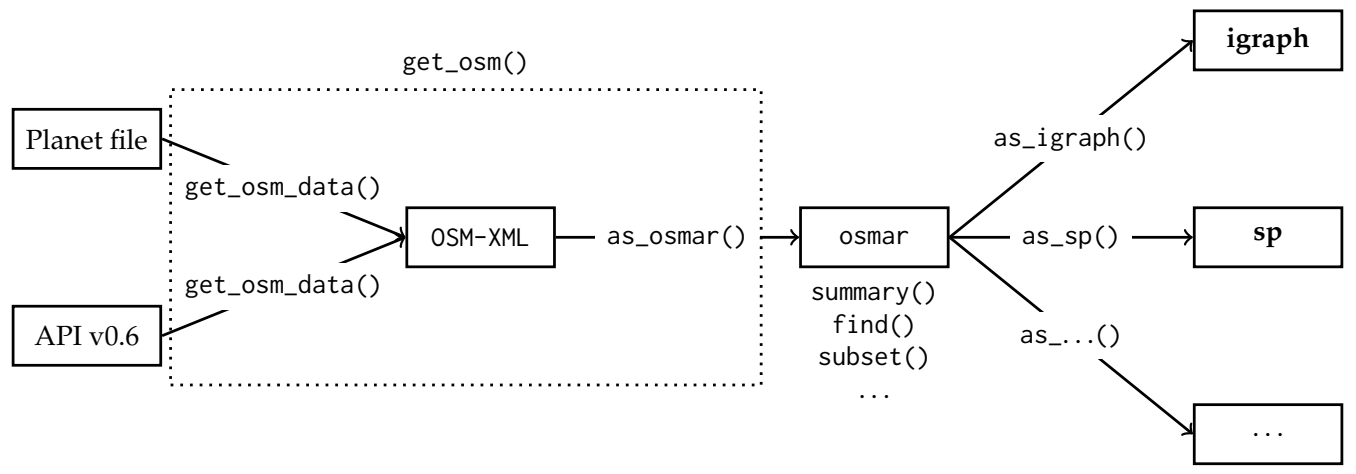

Figure 1: Schematic description of the osmar concept.

\section{Getting the data}

We begin with defining the data source. Currently two sources, HTTP-API and planet files, are supported. In this section we use the API of the OSM project with the default URL url = http: //api.openstreetmap.org/api/0.6/:

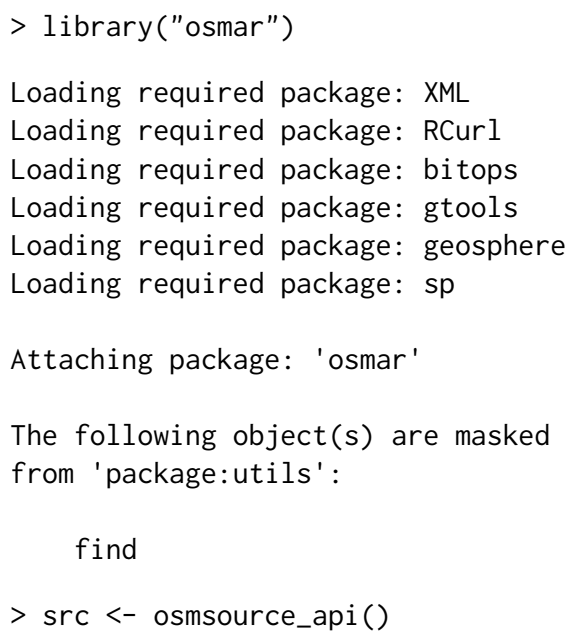

We can retrieve elements by using the IDs of the elements. The IDs in these examples have been extracted by hand from the OpenStreetMap website (via its export functionality). For example, one node:

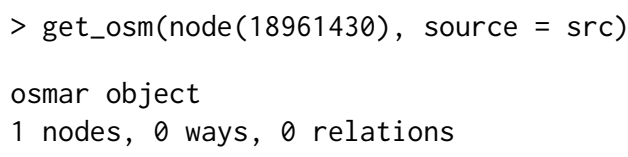

Or, one way with the way-related data only or with the data for all referenced elements (nodes and relations):

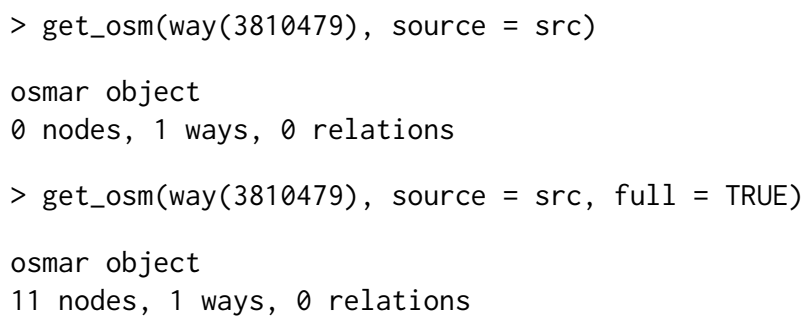

The first statement retrieves the way only (because the default value of the full argument is FALSE). The second statement additionally retrieves all nodes that are members of the way (i.e., all nodes that define the way).

The second possibility to retrieve elements is to specify a bounding box by defining the left, bottom, right, and top coordinates (corner_bbox ()$)$, or the center point and width and height in meters (center_bbox()): 


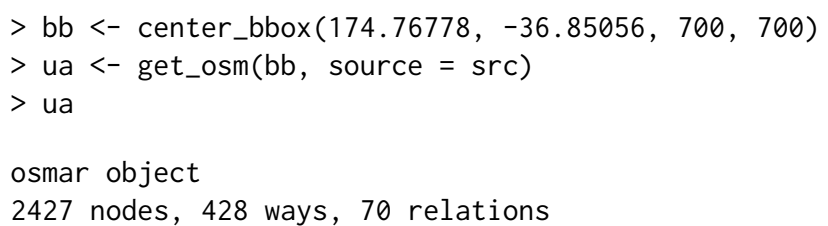

The use of planet files via Osmosis as source works analogously. The source is specified by the function osmsource_osmosis(). The function's two arguments are the path to the planet file (file) and the path to the 'osmosis' tool (osmosis = "osmosis"). Note that per default it is assumed that the Osmosis executable is in your 'PATH' environment variable. The navigator example demonstrates the usage of planet files.

\section{Working with the data}

The retrieved osmar object is a list with the three elements nodes, ways, and relations. Each element again is a list containing data. frames for the attributes (the attrs list element) and meta-data (the tags list element) of the OSM elements. Ways and relations additionally have a data. frame containing their members (the refs list element).

Summarize. For each element nodes, ways, and relations of an osmar object an individual summary method is available. The overall summary method for an osmar object shows the three individual summaries all at once.

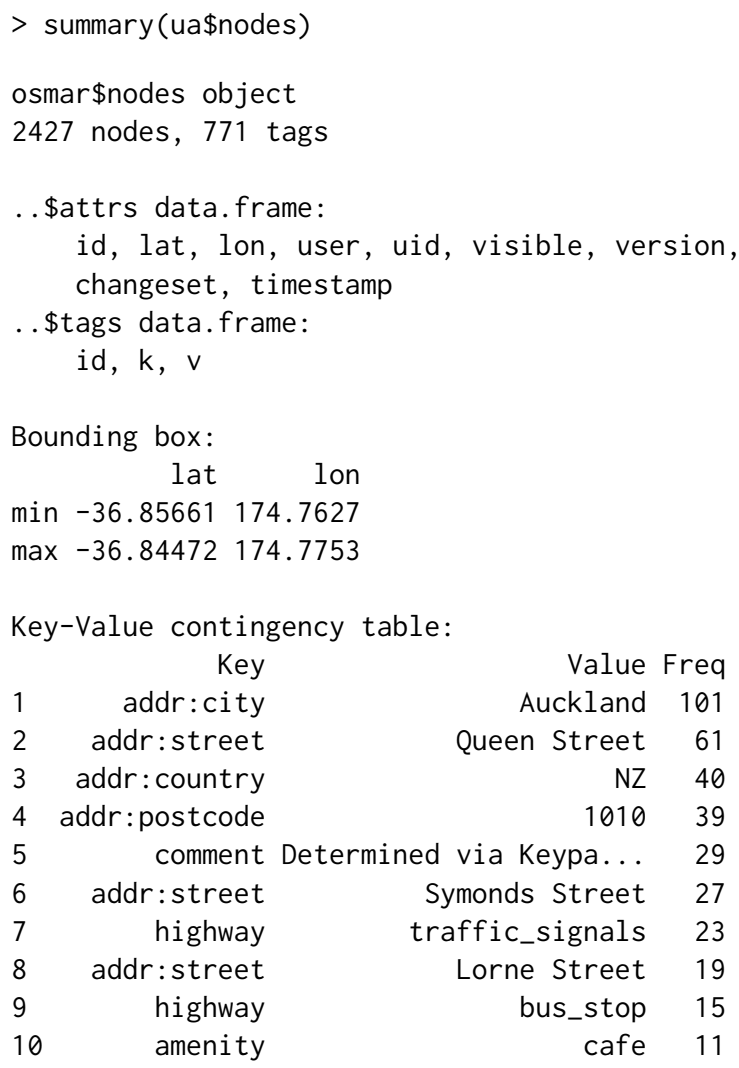

In the case of the summary for nodes, the number of elements and tags, as well as the available variables for each corresponding data. frame are shown. The bounding box of the coordinates and a contingency table of the top ten most frequently available key-value pairs are printed.

The summaries for the other two elements ways and relations are similar. Note that these methods in fact return the contingency table of all available key-value pairs and, in addition, further information which is not printed but may be useful for a descriptive analysis. We refer to the help pages (e.g., ?summary.nodes) for a detailed description of the return values.

Find. In order to find specific elements within the osmar object, the find() function allows the object to be queried with a given condition. As the basis of osmar objects are data.frames, the condition 
principally is a logical expression indicating the rows to keep. In addition, one has to specify to which element (nodes, node(); ways, way(); or relations, relation()) and to which data (attributes, attrs(); meta-data, tags (); or members, refs ()) the condition applies.

If, for example, we want to find all traffic signal nodes, we know from the object's summary that the corresponding value in the attrs data.frame is "traffic_signals". We can express this condition as follows:

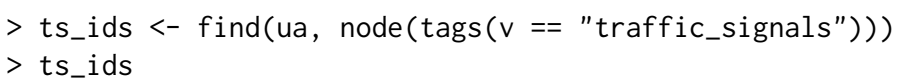

[1] $257696352576963725769641 \ldots$

The result is a vector with node IDs (in this case 25 traffic signal nodes) or NA. If the correct spelling is unknown, the defined binary operators \%agrep\% for approximate matches (see ?agrep) and \%grep\% for pattern matches (see ?grepl) can be used:

$>$ bs_ids <- find(ua, node(tags (v \%agrep\% "busstop")))

$>$ bs_ids

[1] $678301119737159091 \quad 1318401034 \ldots$

This returns 15 bus stops available in the ua osmar object.

We use the functions find_down() and find_up() to find all related elements for given element IDs. The OSM basic elements define a hierarchy,

$$
\text { node } \leftarrow \text { way } \leftarrow \text { relation, }
$$

and these two functions enable us to find the related elements up and down the hierarchy. For example, find_up() on a node returns all related nodes, ways, and relations; find_down() on a node returns only the node itself. On the other hand, find_up() on a relation returns only the relation itself; find_down() on a relation returns the relation and all related ways and nodes.

$>$ hw_ids <- find(ua, way (tags $(k==$ "highway")))

$>$ hw_ids <- find_down(ua, way(hw_ids))

In this example we find all ways that have a tag with the k attribute set to "highway". These contain hardened and recognised land routes between two places used by motorised vehicles, pedestrians, cyclists, etc. The return value of find_down() and find_up () is a list containing the element IDs:

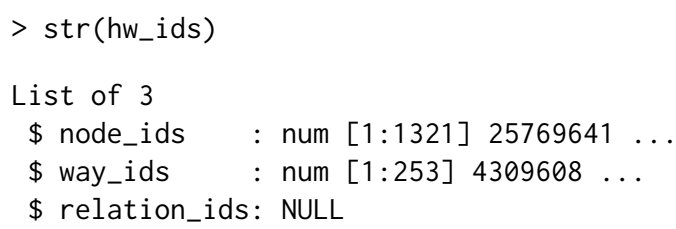

Subset. The return value of the find functions then can be used to create subsets of osmar objects. The subset () method for osmar objects takes element IDs and returns the corresponding data as osmar objects. For example, the two subsets based on the traffic signal and bus stop element IDs are:

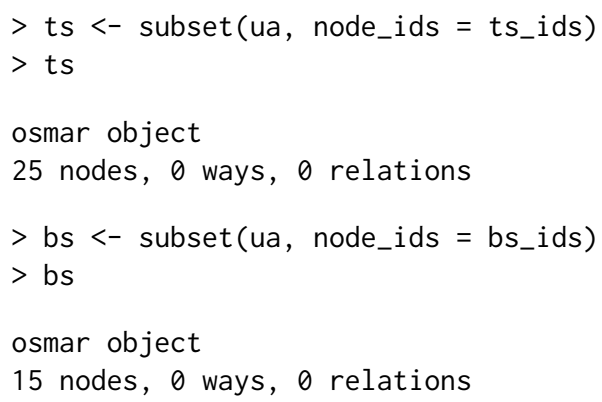

The subset based on the highway element IDs is:

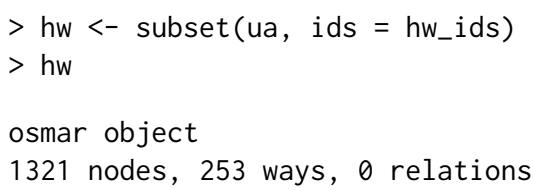


Note that the subsetting of osmar objects is divided into the two steps "finding" and "subsetting" to have more flexibility in handling the related elements (here with using find_down() and find_up(), but more sophisticated routines can be imagined).

Plot. The visualization of osmar objects is possible if nodes are available in the object (as only these OSM elements contain latitude and longitude information). The functions plot_nodes() and plot_ways() plot the available nodes as dots and ways as lines, respectively. The plot() method combines these two function calls. Note that this is a plot of the raw data and no projection is done (see the following section for a projected visualization).
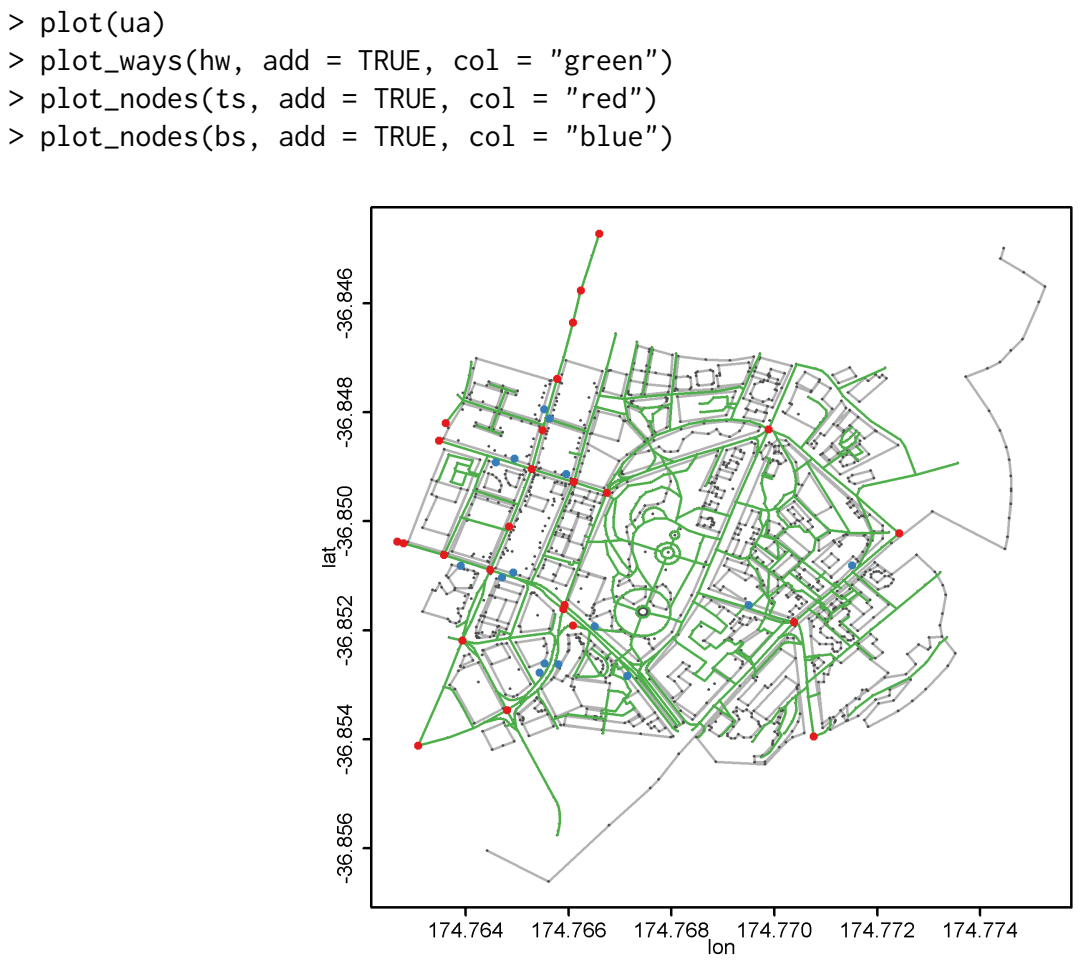

Figure 2: University of Auckland; roads are green lines; bus stops are blue and traffic signals are red points.

\section{Converting the data}

In order to use the complete power of R on OpenStreetMap data, it is essential to be able to convert osmar objects into commonly used objects based on classes provided by other packages. Currently, osmar provides two converters - into the sp (Bivand et al., 2008) and the igraph (Csardi, 2011) packages. In this section we show the conversion to sp objects, the navigation device example shows the conversion to igraph objects.

The sp package provides special data structures and utility functions for spatial data. Spatial data classes are available for points, lines, and polygons and others (see Bivand et al., 2008). The osmar package provides the as_sp() function,

$>\operatorname{args}($ as_sp)

function(obj, what $=c($ "points", "lines", "polygons"),

crs $=$ osm_crs () , simplify $=$ TRUE)

NULL

to convert an osmar object into the corresponding classes for points, lines, and polygons in the sp package (given the required data are available). Note that the appropriate WGS84 coordinate reference system (CRS) for OpenStreetMap data is used (cf. osm_crs()).

Polygons. Polygons are used to represent areas, which OpenStreetMap represents as closed ways. Buildings, for example, are closed ways and can be converted to an sp polygon object: 
$>$ bg_ids <- find(ua, way (tags $(k==$ "building")))

$>$ bg_ids <- find_down (ua, way(bg_ids))

$>$ bg $<-$ subset (ua, ids = bg_ids)

$>$ bg

osmar object

991 nodes, 110 ways, 0 relations

$>$ bg_poly <- as_sp(bg, "polygons")

The result is a SpatialPolygonsDataFrame with the osmar object's attributes (the attrs element) as its data. Functionality provided by the sp package can now be used to analyze the OSM data; e.g., the summary() method or the spplot() method-the latter one, for example, to see how often each building was modified:

> spplot(bg_poly, c("version"))

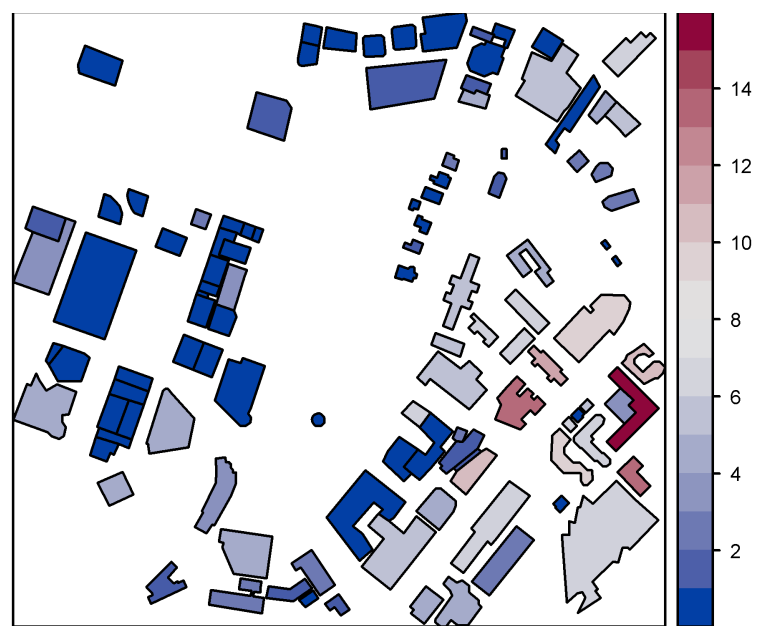

Figure 3: Number of modifications per building.

Meta-data (the tags element) and members (the refs element) are not automatically converted to a SpatialPolygonsDataFrame's data.

Lines and points. The conversion of lines and points works similarly:

$>$ hw_line <- as_sp(hw, "lines")

$>$ bs_points <- as_sp(bs, "points")

The results are SpatialLinesDataFrame and SpatialPointsDataFrame objects, respectively.

In order to finalize the University of Auckland example we create a bus route map and visualize the available bus routes belonging to the bus stops. Therefore, we find all bus relations available in the object, retrieve the corresponding data from the OSM API, and convert the data into lines (note that this computation takes some time):

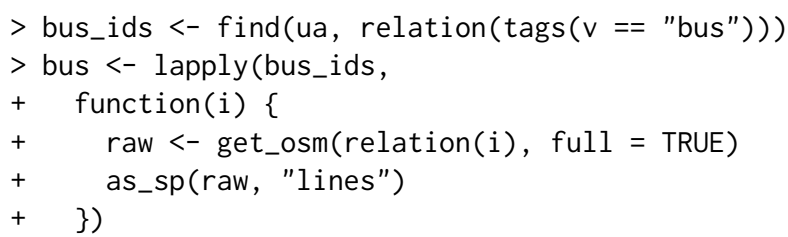

We use the argument full = TRUE to retrieve the relation itself and all related members. In detail, this means we retrieve all nodes, ways, and relations that are members of the specified relation; and, recursively, all nodes that are members of the retrieved ways.

We then use the sp plot methods to create the final bus route map:

$>$ plot(bg_poly, col = "gray")

$>$ plot (hw_line, add $=$ TRUE, col $=$ "green") 

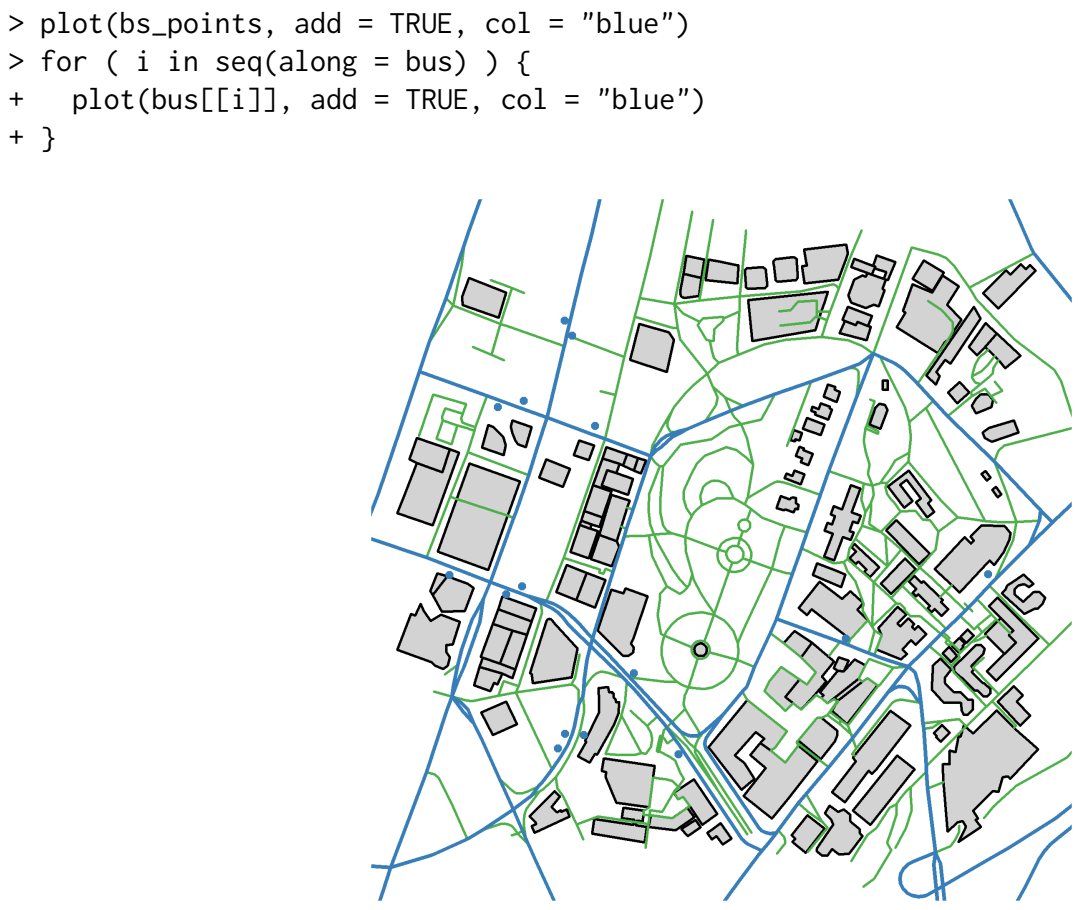

Figure 4: Bus route map of the University of Auckland; roads are green lines; bus stops and bus routes are blue points and lines

\section{$R$ as navigator}

We always wanted to know how a navigation device works. Now with osmar, R provides the necessary components and this serves as nice example on how to use osmar. The general idea is to (1) get the data, (2) extract all highways, (3) create a graph of all highway nodes with the distance between the highway nodes as edge weights, (4) compute the shortest path on the graph, and (5) trace the path on the highways.

Get the data. We use a planet file from Munich as the data source and use Osmosis (Henderson, 2011) to process the data. Note that 'osmosis' has to be in your 'PATH' environment variable.

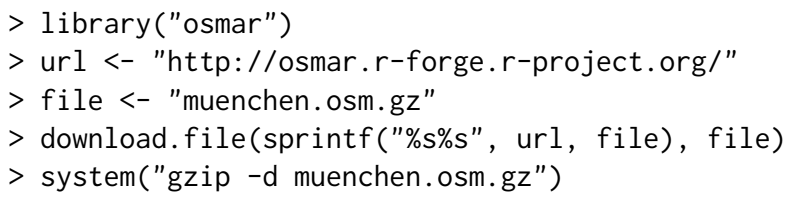

Get the center of Munich with a $3 \mathrm{~km} \times 3 \mathrm{~km}$ bounding box:

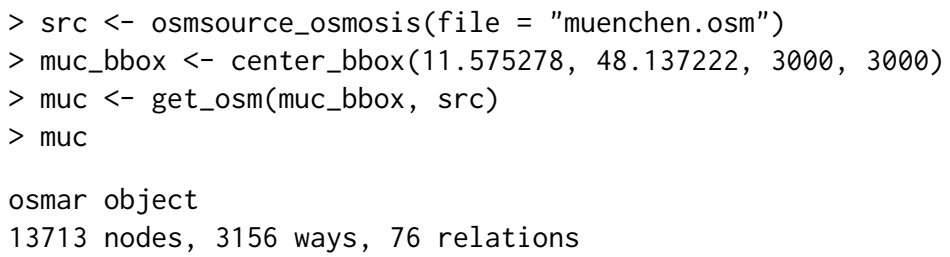

For the navigation device we only need streets. This means, we have to find all ways that are tagged as highways and have a name tag, then find the associated nodes, and finally subset the full osmar object:

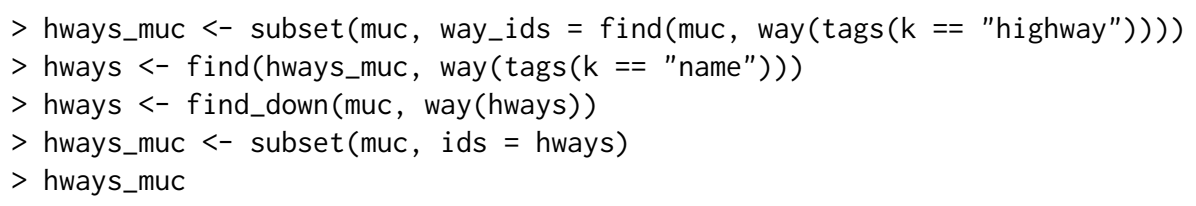


osmar object

3889 nodes, 995 ways, 0 relations

Suppose we want to start our route at the "Sendlinger Tor". This means we first have to find a node that is tagged with the name "Sendlinger Tor" and then the nearest highway node:

$>$ hway_start_node $<-$ local $(\{$

$+\quad$ id $<-$ find(muc, node(tags $(v==$ "Sendlinger Tor")))[1]

+ find_nearest_node(muc, id, way $(\operatorname{tags}(k==$ "highway")))

$+\}$ )

$>$ hway_start <- subset(muc, node(hway_start_node))

For a given node (by its ID), the function find_nearest_node() finds the nearest node with the specified conditions (the package geosphere, Hijmans et al., 2011 is used to compute the distances). The end of the route should be in the northeast part of Munich; so we find nodes that are in the northeast and take one highway node:

$>$ hway_end_node $<-$ local $(\{$

$+\quad$ id <- find(muc, node $(\operatorname{attrs}($ lon $>11.59 \&$ lat $>48.150)))[1]$

+ find_nearest_node(muc, id, way $(\operatorname{tags}(k==$ "highway")))

+ \})

$>$ hway_end <- subset(muc, node(hway_end_node))

Finally, we visualize the initial situation:

$>$ plot_nodes(muc, col = "gray")

$>$ plot_ways (hways_muc, add $=$ TRUE)

$>$ plot_nodes(hways_muc, add $=$ TRUE, col = "black")

$>$ plot_nodes (hway_start, add $=$ TRUE, col = "red")

$>$ plot_nodes (hway_end, add = TRUE, col = "blue")

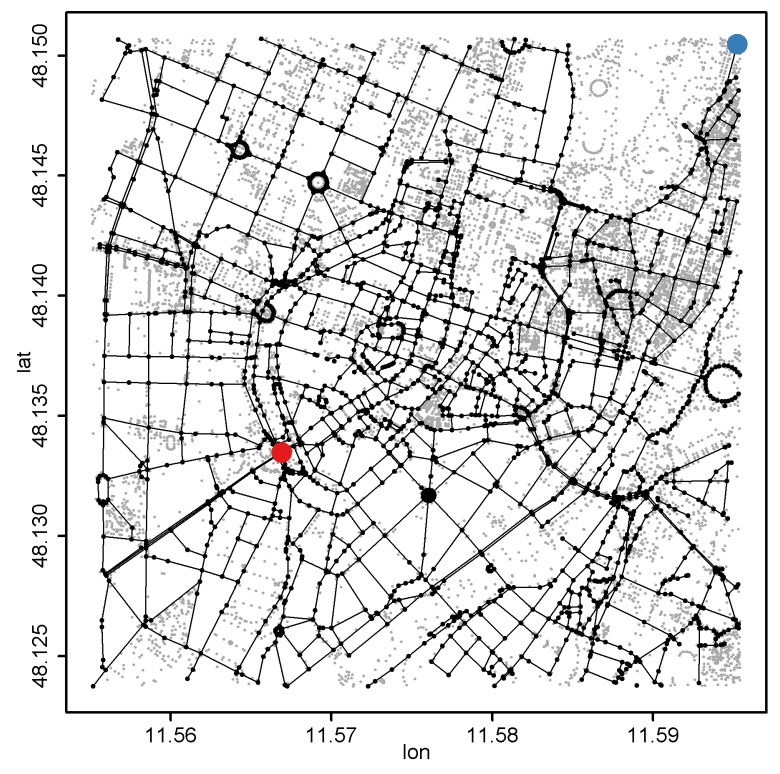

Figure 5: Highway map of Munich center.

The gray nodes are all nodes available in the full osmar object, the black nodes and lines are the road networks available in the highway-osmar object, the red and blue dots denote the starting and ending nodes of the searched route.

Compute the route. In order to compute the shortest route between the defined starting and ending nodes, we convert the highway-osmar object into a graph. R provides a set of packages to work with graphs, we decided to use igraph:

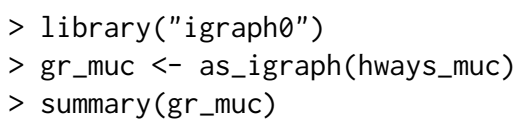


Vertices: 2381

Edges: 2888

Directed: TRUE

No graph attributes.

Vertex attributes: name.

Edge attributes: weight, name.

The osmar object nodes define the nodes of the graph (node IDs are used as graph node names). The osmar object ways define the edges (way IDs are used as edge names), and the weights of the edges are the geographical distance between the nodes.

The igraph package provides different shortest path algorithms (e.g., Dijkstra and Bellman-Ford) via the function get. shor test. paths(). The shortest route (not necessarily unique) is:

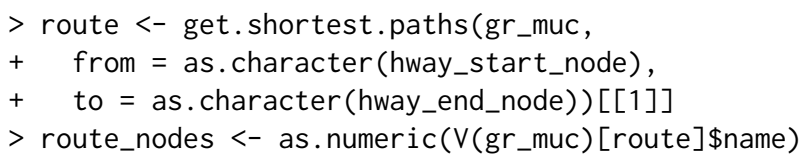

We construct a new osmar object containing only elements related to the nodes defining the route:

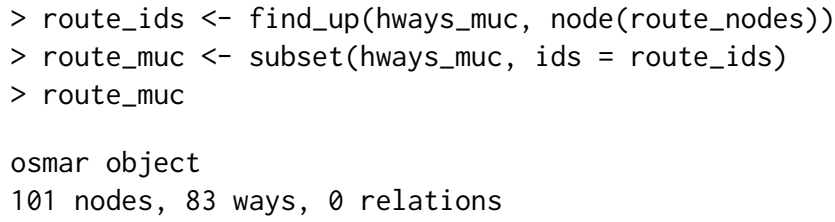

And add the route to the highway map of Munich center in Figure 5:

$>$ plot_nodes(route_muc, add = TRUE, col = "green")

$>$ plot_ways (route_muc, add = TRUE, col = "green")

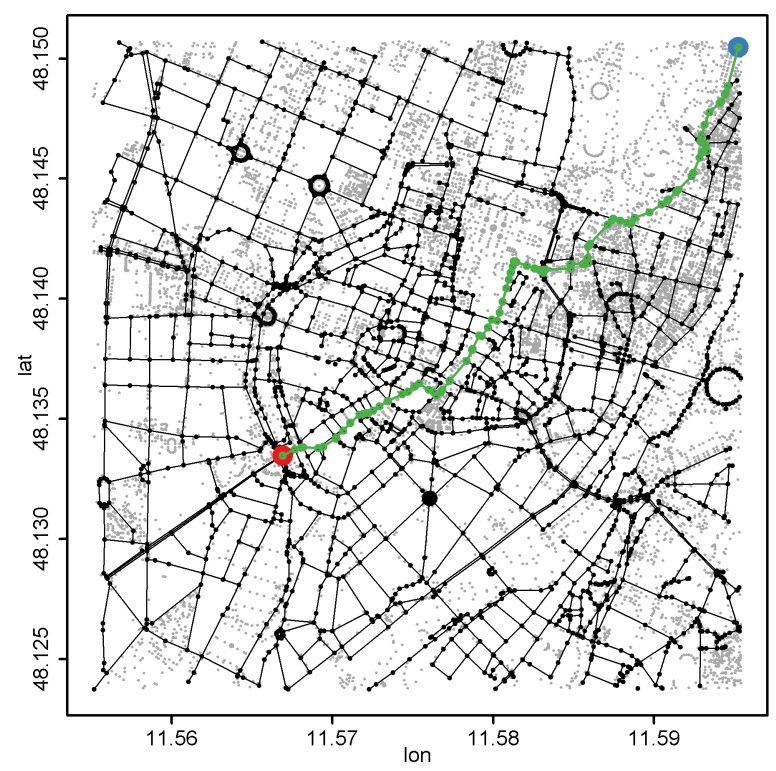

Figure 6: Shortest route on highway map of Munich center.

Route details. In order to present route details like street names, distances, and directions we have to work directly on the internals of the osmar objects.

We start by extracting the route's node IDs (which are in the correct order) and the way IDs (which we have to order) where the nodes are members:

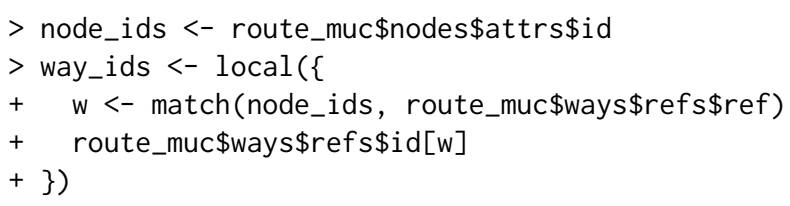


Then we extract the names of the ways in the correct order:

$>$ way_names <- local $(\{$

$+\mathrm{n}<-$ subset(route_muc\$ways\$tags, k == "name")

$+\mathrm{n}[\mathrm{match}($ way_ids, $\mathrm{n} \$ \mathrm{id})$, , $\mathrm{v} "]$

$+\})$

The next step is to extract the nodes' coordinates,

$>$ node_coords <- route_muc $\$$ nodes $\$$ attrs $[$, c("lon", "lat")]

and to compute the distances (meters) and the bearings (degrees) between successive nodes (using the package geosphere):

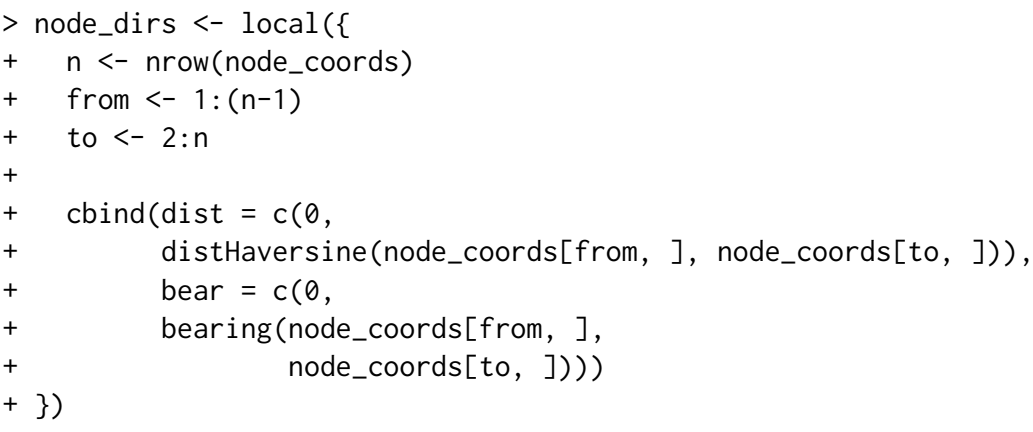

Finally, we pack together all the information, and additionally compute the cumulative distance and a 16-point compass rose direction (the compass () function is available in the "navigator" demo from the osmar package):

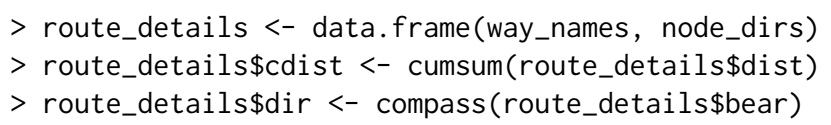

The result is a data. frame with a row for each node of the route. The row shows the name of the associated way, the distance (meters) and bearing (degrees and compass rose) to the successive node of the route, and the cumulative distance:

$>$ head(route_details)

$\begin{array}{rrrrrr} & & \text { way_names } & \text { dist } & \text { bear } & \text { cdist dir } \\ 1 & \text { Sendlinger-Tor-Platz } & 0 & 0 & 0 & \text { N } \\ 2 & \text { Wallstraße } & 65 & 62 & 65 \text { ENE } \\ 3 & \text { Herzog-Wilhelm-Straße } & 29 & 75 & 94 \text { ENE } \\ 4 & \text { Oberanger } & 10 & 78 & 104 \text { ENE } \\ 5 & \text { Oberanger } & 69 & 94 & 173 & \text { E } \\ 6 & \text { Nikolaus-Gradl-Weg } & 25 & 76 & 198 \text { ENE }\end{array}$

Needless to say that this navigation device can be made much more sophisticated. The osmar package contains the complete source code of the basic navigation device as a demo and we invite everybody to improve $R$ as a navigator.

\section{Summary}

The osmar package extends the R ecosystem with infrastructure to work together with the OpenStreetMap project. So far, functionality is available to get data from different sources (e.g., planet file and API v0.6), to consolidate the data as an R osmar object, to work with the osmar object (e.g., subsetting and plotting), and to convert it to objects based on classes provided by other packages (e.g., to igraph and sp objects).

Future work includes the implementation of further converters; e.g., a converter from osmar objects to raster image objects via the OpenStreetMap package. We are also interested in implementing converters from objects provided by other $\mathrm{R}$ packages to osmar objects and in saving these objects in different OpenStreetMap sources via a put_osm() function. This would be, in fact, the completion of the osmar concept illustrated in Figure 1 with arrows from the right to the left blocks.

Furthermore, we would like to incorporate tools originated in the OpenStreetMap ecosystem. One idea is the implementation of a rule-based rendering tool for generating SVG images of OSM data along the lines of Osmarender (Topf, 2011). Another interesting project is Osmium, a fast and flexible 
C++ and Javascript toolkit and framework for working with OSM data (Topf, 2012). An R interface (potentially via Rcpp modules; Eddelbuettel and François, 2011) would provide a very fast and flexible way to work with large OSM data sets.

\title{
Acknowledgment
}

The authors thank two anonymous reviewers and Joe Sakshaug for their constructive comments to improve the manuscript.

\section{Bibliography}

R. S. Bivand, E. J. Pebesma, and V. Gomez-Rubio. Applied Spatial Data Analysis with R. Springer, NY, 2008. URL http://www. asdar-book.org/. [p57]

G. Csardi. igraph: Network Analysis and Visualization, 2011. URL http://cran.r-project.org/ package=igraph. R package version 0.5.5-2. [p57]

D. Eddelbuettel and R. François. Rcpp: Seamless R and C++ integration. Journal of Statistical Software, 40(8):1-18, 2011. URL http: //www. jstatsoft.org/v40/i08/. [p63]

I. Fellows. OpenStreetMap: Access to OpenStreetMap Raster Images, 2012. URL http://CRAN.Rproject. org/package=0penStreetMap. R package version 0.2. [p53]

B. Henderson. osmosis, 2011. URL http://wiki openstreetmap.org/wiki/Osmosis. Java application version 0.39. [p53,59]

R. J. Hijmans, E. Williams, and C. Vennes. geosphere: Spherical Trigonometry, 2011. URL http: //CRAN.R-project.org/package=geosphere. R package version 1.2-24. [p60]

D. Kahle and H. Wickham. ggmap: A Package for Spatial Visualization with Google Maps and OpenStreetMap, 2012. URL http://CRAN. R-project. org/package=ggmap. R package version 2.1. [p53]

M. Loecher. RgoogleMaps: Overlays on Google Map Tiles in R, 2012. URL http: //CRAN. R-project. org/package=RgoogleMaps. R package version 1.2.0. [p53]

OSM Foundation. The OpenStreetMap Project, 2011. URL http: //openstreetmap.org. [p53]

T. Schlesinger and M. J. A. Eugster. osmar: OpenStreetMap and R, 2012. URL http: //cran. r-project. org/package=osmar. R package version 1.1. [p53]

J. Topf. osmarender, 2011. URL http://wiki. openstreetmap.org/wiki/Osmarender. XSLT transformation scripts. [p62]

J. Topf. Osmium, 2012. URL http://wiki.openstreetmap.org/wiki/Osmium. Fast and flexible C++ and Javascript toolkit and framework for working with OSM data. [p63]

\author{
Manuel J. A. Eugster \\ Institut für Statistik \\ Ludwig-Maximilians-Universität München \\ Ludwigstrasse 33, 80539 Munich \\ Germany \\ manuel. eugster@stat.uni-muenchen.de \\ Thomas Schlesinger \\ Institut für Statistik \\ Ludwig-Maximilians-Universität München \\ Ludwigstrasse 33, 80539 Munich \\ Germany \\ tho.schlesinger@googlemail.com
}

